\title{
Scintillation and beam-wander analysis in an optical ground station-satellite uplink
}

\author{
Federico Dios, Juan Antonio Rubio, Alejandro Rodríguez, and Adolfo Comerón
}

\begin{abstract}
In an optical communication link between an optical ground station and a geostationary satellite the main problems appear in the uplink and are due to beam wander and to scintillation. Reliable methods for modeling both effects simultaneously are needed to provide an accurate tool with which the robustness of the communication channel can be tested. Numerical tools, especially the split-step method (also referred to as the fast-Fourier-transform beam propagation method), have demonstrated their ability to deal with problems of optical propagation during atmospheric turbulence. However, obtaining statistically significant results with this technique is computationally intensive. We present an analyticalnumerical hybrid technique that provides good information on the variance in optical irradiance with an important saving of time and computational resources. (C) 2004 Optical Society of America

OCIS codes: $\quad 010.1300,010.1330,010.3310$.
\end{abstract}

\section{Introduction}

The intrinsic turbulence of the atmosphere represents the main obstacle to setting reliable optical communication channels both for horizontal paths and for those designed to link with geostationary or other types of satellite. The first effect to take into account is scintillation, which by nature is the same effect that one observes in looking at the stars at night or looking at city lights from a certain distance away. The twinkling of the light is due to smallscale eddies formed in the atmosphere. The scintillation phenomenon has been the object of an important body of work. ${ }^{1-11}$

Atmospheric turbulence has also another dimension, which may be considered the typical maximum size of individual eddies formed in the atmosphere. The larger eddies, which are crossed by the entire optical beam, cause some degree of refraction, and thus the beam deviates slightly from its original path. This phenomenon is known as beam wander.5,10,12

Beam wander is negligible in the downlink from satellites. This is so because turbulence eddies

The authors are with the Department of Signal Theory and Communications, Universitat Politècnica de Catalunya, C/Sor Eulalia d’Anzizu s/n. Campus Nord, 08034-Barcelona, Spain. F. Dios's e-mail address is fede@tsc.upc.es.

Received 7 August 2003; revised manuscript received 3 February 2004; accepted 2 April 2004.

0003-6935/04/193866-08\$15.00/0

(C) 2004 Optical Society of America much smaller than the beam size do not displace the beam's centroid significantly, and the downlink optical beam arrives at the atmosphere with a width much larger than any turbulent eddy. Instead, the wavefront tilt at the receiver produced by the atmospheric turbulence gives rise to the so-called angleof-arrival fluctuations. , $^{2,5-7,10}$

The situation is different in the uplink. The beam width remains smaller than the outer scale of the turbulence during its atmospheric path, and the beam may leave the atmosphere with a significant change in direction. An analysis of the variations in optical irradiance that are due to the combined effect of scintillation and beam wander is the subject of this research.

We have made use of existing models and approximate expressions. The atmospheric air mass that is traversed in a typical ground-satellite link (in the direction of the zenith) is equivalent to that encountered in a few-hundred-meter-long horizontal ground-level path. Then we can expect weak or moderately strong turbulence, for which available models are sufficiently accurate.

We briefly present the theoretical results that we used in our simulations. Although in this paper we limit our simulation results to collimated beams, the method could easily be extended to converging or diverging beams, as the subject theory may be generalized to these other cases.

\section{Rytov's Theory and Beam Wander}

At this point it is necessary to clarify what the relationship is between the Rytov approximation and the 
beam-wander phenomenon. In the earliest analysis of optical propagation through atmospheric turbulence, as the problem was solved for plane and spherical waves, such a question did not exist. In the first works that dealt with Gaussian beams, such as those of Schmeltzer ${ }^{13}$ and Ishimaru, ${ }^{14}$ the Rytov method was retained as the basic tool of the analysis, although it was not clear whether the beam-wander effect was actually considered within the model. In fact, to our knowledge this point has not been resolved to date; the scientific community has not posed it explicitly as a question to be clarified. In our experience it seems that most researchers have assumed as a matter of fact that beam wander is in some way included within analyses following Rytov, whereas others maintain doubt that this is so. ${ }^{15}$

At a first glance there is a primary and strong objection to accepting that the displacement of the beam as a whole can be appropriately modeled by Rytov's theory: The reason is that the final beam that reaches the receiver plane is considered the diffracted, unperturbed beam plus several minor terms. It is clear that in each particular instance of the experiment of launching a laser beam through turbulence the weakly perturbed field cannot reproduce the real beam because that beam can be tilted considerably in an arbitrary direction, even with weak or moderately strong turbulence. Things become more complicated if someone argues that a single instance is not so important because we are interested primarily in the statistical, long-term effect. As a consequence, and given that the beam wander tends to cancel itself on average, the Rytov technique or any other perturbative technique could validly deal with propagation problems, including beam wander. In this respect we are still not in a position to give a definitive answer, however, several conclusions can be drawn:

(i) Hufnagel ${ }^{16}$ mentioned some discrepancies between theory and experiment in propagation of Gaussian beams through turbulence, and he concluded that perhaps this is not surprising because, presumably, beam-wander effects are not accounted for in the Rytov approximation.

(ii) Titterton ${ }^{17}$ was the first author, to our knowledge, who dealt with the scintillation and beamwander phenomena simultaneously and, in fact, as two independent effects. He showed that the power fluctuations caused by beam tilt can be substantially greater than those caused by scintillation.

(iii) As is well known, formulations proposed for the characterization of beam wander,18,19 as it occurs with fluctuations in the angle of arrival in the downlink, are deduced through physical considerations and mathematical tools that are entirely different from Rytov or other perturbative techniques. So it is implicitly recognized that no perturbative technique can treat such a phenomenon as beam wander.

(iv) Finally, the statement that the Rytov method in some way contains the beam-wander effect could perhaps be accepted as a hypothesis in the long-term analysis, assuming that the average beam motion cancels. However, there are at least two important difficulties to be solved: the first is that it should be proved that the statistical nature of atmospheric turbulence effectively forces cancellation of the average beam wander in any situation; the second is that it must be determined to what point or under what conditions beam wander is included in the Rytov or other perturbative technique.

In this study we started from the negative position; that is, that the beam-wander phenomenon has to be added explicitly to the Rytov approximation or to any other formulation based on the Rytov approximation. Our numerical calculations seem to confirm this point of view, at least for uplink, in which the beam is traveling through increasingly weaker atmospheric turbulence.

\section{Beam Effects}

The most important part of the scientific literature devoted to optical propagation in atmospheric turbulence has been focused on the scintillation phenomenon. The classic models used for describing the propagation of optical radiation are plane and spherical waves. The finite transverse dimensions of real laser beams require that other phenomena in addition to scintillation must to be taken into account.

There are three characteristic magnitudes to be considered in beam propagation through a turbulent medium. These are short exposure, or short-term average beam width; long exposure, or long-term beam width; and beam wander. ${ }^{5}$ On the assumption of weak turbulence, it is possible to find approximate analytical expressions for all three quantities.

The first effect of turbulence on the optical beam is that diffraction seems to be somewhat larger than that which occurs in free space. As a consequence, the average physical width, or short-term beam width, is larger than that predicted by the theory of Gaussian beams. The second effect is the beam wandering described above. The centroid of the spot dances about the vacuum propagation center in the reception plane. The random displacement of the spot centroid appears to follow a Rayleigh distribution of probability. ${ }^{5}$

The resultant long-term spot size becomes a superposition of the instantaneous spots that reach the receiver. This long-term spot represents the average field or irradiance in the statistical problem, and it will tend, theoretically, to a Gaussian distribution. The following relation ${ }^{5,6,10}$ is well known:

$$
W_{\mathrm{LT}}^{2}(z)=W_{\mathrm{ST}}^{2}(z)+2\left\langle\beta^{2}\right\rangle,
$$

where $W_{\mathrm{LT}}(z)$ and $W_{\mathrm{ST}}(z)$ are the long-term and the short-term beam widths, respectively, $z$ is the propagation direction, and $\left\langle\beta^{2}\right\rangle$ is the second-order moment of the beam displacement. In this paper we define the beam width as the width for which the electric field decays by $1 / e$ with respect to its maximum. 


\section{A. Long-Term Beam Spread}

It has been shown ${ }^{5}$ that the average irradiance width in a long exposure may be obtained, for collimated beams, as

$$
W_{\mathrm{LT}}^{2}(z=L)=W_{0}^{2}\left(1+\frac{L^{2}}{Z_{0}^{2}}\right)+2\left(\frac{4 L}{k_{0} r_{0, s}}\right)^{2},
$$

where $W_{0}$ is the beam waist at the origin, $k_{0}$ is the wave number, $Z_{0}$ is the Rayleigh distance, $L$ is the propagated distance to the receiver, and $r_{0, s}$ is the coherence diameter for spherical waves in the turbulent atmosphere. The last-named parameter gives a measure of the strength of the turbulence, and it is written as $^{5}$

$$
r_{0, s}=\left[0.42 k^{2} \int_{0}^{L} C_{n}{ }^{2}(z)\left(\frac{L-z}{L}\right)^{5 / 3} \mathrm{~d} z\right]^{-3 / 5}
$$

for the uplink.

$C_{n}{ }^{2}(z)$ is the structure constant of the refractiveindex distribution, which diminishes with height. For this function several parametric models have been proposed, and it may be measured in each specific site with the appropriate experimental setups. ${ }^{6,10,16}$ Andrews et al. ${ }^{20}$ gave another expression for the long-term beam width that was derived from the second-order Rytov approximation ${ }^{21}$ :

$$
W_{\mathrm{LT}}(L)=W(L) \sqrt{1+G_{u}},
$$

where $W(L)$ is the diffraction-limited beam width in free space and

$$
\begin{aligned}
G_{u}= & 4 \pi^{2} k_{0}{ }^{2} \int_{0}^{L} \int_{0}^{\infty} \kappa \Phi_{n}(z, \kappa) \\
& \times\left\{1-\exp \left[-\frac{\Lambda L \kappa^{2}}{k_{0}}\left(1-\frac{z}{L}\right)^{2}\right]\right\} \mathrm{d} \kappa \mathrm{d} z,
\end{aligned}
$$

where $\Phi_{n}(z, \kappa)$ is the refractive-index power spectrum, $\kappa$ is the spatial frequency, and $\Lambda$ is a beam parameter in the form

$$
\Lambda=\frac{2 L}{k_{0} W^{2}(L)} .
$$

In our calculations we tested both Eqs. (2) and (4), and the results were similar, as we show in Section 6 below.

\section{B. Short-Term Beam Spread}

It is common to accept the approximate expression derived by Yura for the short-term beam spread, ${ }^{22}$ $W_{\mathrm{ST}}$ :

$$
\begin{aligned}
W_{\mathrm{ST}}^{2}(z=L)= & W_{0}^{2}\left(1+\frac{L^{2}}{Z_{0}^{2}}\right)+2 \\
& \times\left\{\frac{4.2 L}{k r_{0, s}}\left[1-0.26\left(\frac{r_{0, s}}{W_{0}}\right)^{1 / 3}\right]\right\}^{2},
\end{aligned}
$$

which is valid when the condition $0.26\left(r_{0, s} / W_{0}\right)^{1 / 3} \ll$ 1 is satisfied. Sometimes the factor of 4.2 in Eq. (7) is approximated by 4 ; in fact, the results are nearly identical.

\section{Beam Wander}

The random displacements of the centroid of the propagated beam along the $X$ and $Y$ coordinates, $\beta_{x}$ and $\beta_{y}$, are normal random variables, with zero mean and equal standard deviation. ${ }^{5}$ We have

$$
{\sigma_{\beta_{X}}}^{2}={\sigma_{\beta_{Y}}}^{2}=\left\langle\beta_{x}{ }^{2}\right\rangle=\left\langle\beta_{y}{ }^{2}\right\rangle .
$$

The relations to the absolute displacement of the centroid, however, are

$$
\begin{aligned}
\beta & =\sqrt{{\beta_{x}{ }^{2}+\beta_{y}{ }^{2}}^{2}} \\
\left\langle\beta_{x}{ }^{2}\right\rangle & =\left\langle\beta_{y}{ }^{2}\right\rangle=1 / 2\left\langle\beta^{2}\right\rangle .
\end{aligned}
$$

The approximate expression for the second-order moment, assuming that the turbulence is weak and that the beam maintains its Gaussian profile, is ${ }^{23}$

$$
\left\langle\beta^{2}\right\rangle=2.07 \int_{0}^{L} C_{n}{ }^{2}(z)(L-z)^{2}\left[\frac{1}{W_{s}(z)}\right]^{1 / 3} \mathrm{~d} z .
$$

\section{Scintillation}

From the earlier works of Tatarski ${ }^{2}$ an important theoretical and experimental body of work has been developed in the past several decades. As was said above, most of the theory that has been developed was devoted to plane and spherical optical waves. The first studies that dealt with scintillation in laser beams are those due to Schmeltzer ${ }^{13}$ and to Ishimaru. ${ }^{14}$

We use the expressions derived later by Andrews et al. ${ }^{20}$ Although we follow closely the study described in that reference, we rewrite some of the expressions to avoid confusion, because there are some errata and mathematical errors in the original work. By "scintillation" we shall understand the fluctuations of optical power received by a point photodetector after the wave has crossed over a turbulent medium. Usually that term is used, however, to define irradiance fluctuations, in which the features of a particular photodetector, especially the size of the reception area, are not taken into account.

Scintillation is measured by means of the so-called scintillation index, that is, the irradiance variance normalized to mean unity. Then its general expression is

$$
\sigma_{I}^{2}=\frac{\left\langle I^{2}\right\rangle-\langle I\rangle^{2}}{\langle I\rangle^{2}},
$$

where $I$ is the irradiance at some point in the detector plane.

The scintillation index for a laser beam at a certain distance $L$ can be divided into two terms: the first is the scintillation on axis, at the center point of the beam, and the second takes into account the depen- 
dence of the scintillation on the distance to the center of the spot. ${ }^{20}$ Then we have

$$
\sigma_{I}^{2}(r, L)=\sigma_{I}^{2}(0, L)+\sigma_{I, r}^{2}(r, L) .
$$

The expression for the on-axis scintillation is

$$
\begin{aligned}
\sigma_{I}^{2}(0, L)= & 8 \pi^{2} k_{0}^{2} \int_{0}^{L} \int_{0}^{\infty} \kappa \Phi_{n}(z, \kappa) \\
& \times \exp \left[-\frac{\Lambda L \kappa^{2}}{k_{0}}\left(\frac{L-z}{L}\right)^{2}\right] \\
& \times\left(1-\cos \left\{\frac{L \kappa^{2}}{k_{0}}\left(\frac{L-z}{L}\right)\right.\right. \\
& \left.\left.\times\left[\Theta+\frac{(1-\Theta) z}{L}\right]\right\}\right) \mathrm{d} \kappa \mathrm{d} z
\end{aligned}
$$

where $\Phi_{n}(z, \kappa)$ is the spatial power spectrum of the refractive-index fluctuations, $\kappa$ is the spatial frequency, $k_{0}$ is the wave number in free space, and $\Lambda$ and $\Theta$ are parameters of the laser beam:

$$
\Lambda=\frac{2 L}{k_{0} W^{2}(L)}, \quad \Theta=\left[1+\left(\frac{L}{Z_{0}}\right)^{2}\right]^{-1},
$$

assuming that the beam is initially collimated.

If we take the classic Kolmogorov index power spectrum, $\Phi_{n}(z, \kappa)=0.033 C_{n}{ }^{2}(z) \kappa^{-11 / 3}$, integration in spatial frequency к may be performed, with the result that

$$
\begin{aligned}
\sigma_{I}^{2}(0, L)= & 4 \pi^{2} k_{0}{ }^{2} \Gamma\left(-\frac{5}{6}\right) \times 0.033 \int_{0}^{L} C_{n}^{2}(z) \\
& \times[A(z)]^{5 / 6}\left(1-\left\{1+\left[\frac{B(z)}{A(z)}\right]^{2}\right\}^{5 / 12}\right. \\
& \left.\times \cos \left\{\frac{5}{6} \arctan \left[\frac{B(z)}{A(z)}\right]\right\}\right) \mathrm{d} z
\end{aligned}
$$

where

$$
\begin{aligned}
& A(z)=\frac{\Lambda L}{k_{0}}\left(\frac{L-z}{L}\right)^{2}, \\
& B(z)=\frac{L}{k_{0}}\left(\frac{L-z}{L}\right)\left[\Theta+\frac{(1-\Theta) z}{L}\right] .
\end{aligned}
$$

The scintillation term that depends on the transverse radius in the horizontal plane, $r$, is

$$
\begin{aligned}
\sigma_{I, r}^{2}(r, L)= & 8 \pi^{2} k_{0}{ }^{2} \int_{0}^{L} \int_{0}^{\infty} \kappa \Phi_{n}(z, \kappa) \\
& \times \exp \left[-\frac{\Lambda L \kappa^{2}}{k_{0}}\left(\frac{L-z}{L}\right)^{2}\right] \\
& \times\left\{I_{0}\left[2 \Lambda \kappa r\left(\frac{L-z}{L}\right)\right]-1\right\} \mathrm{d} \kappa \mathrm{d} z,
\end{aligned}
$$

where $I_{0}($ ) is a modified Bessel function of first class and zero order. Again, on the assumption that the turbulence follows the Kolmogorov spectrum, integration may be carried out with respect to $\kappa$, with the result that

$$
\begin{aligned}
\sigma_{I, r}{ }^{2}(r, L)= & 4 \pi^{2} k_{0}{ }^{2} \Gamma\left(-\frac{5}{6}\right) 0.033 \int_{0}^{L} C_{n}{ }^{2}(z)[A(z)]^{5 / 6} \\
& \times\left\{{ }_{1} F_{1}\left[-\frac{5}{6}, 1, \frac{2 r^{2}}{W^{2}(L)}\right]-1\right\} \mathrm{d} z,
\end{aligned}
$$

where ${ }_{1} F_{1}$ is the Kummer confluent hypergeometric function.

\section{Simulation Procedure}

To analyze the behavior of the detected optical signal in the uplink of a ground-satellite channel we have developed a straightforward procedure in which we apply the analytical expressions shown in the preceding sections. The idea is to generate the necessary sequence of random variables to simulate numerically the irradiance seen by a point detector in the satellite.

The irradiance at the receptor plane at any instant of time will have the form

$I(x, y, L)=I_{0} \exp \left[-2 \frac{\left(x-\beta_{x}\right)^{2}+\left(y-\beta_{y}\right)^{2}}{W_{\mathrm{ST}}^{2}(L)}\right]$,

where $\beta_{x}$ and $\beta_{y}$ are normal random variables that define the beam wander. $I_{0}$ will be also a random variable because of scintillation. We can write

$$
I_{0}=\exp [2 \chi(\beta)]
$$

where $\chi(\beta)$ is the (partial) log amplitude of the beam. We use the term "partial" because the Gaussian spatial variation is not included in it. Variable $\chi(\beta)$ takes its dependence on the beam displacement from the variation in scintillation index with the position. We are interested in the study of the irradiance variations at the detector position, in the theoretical center of the beam. So the measured irradiance will be

$$
I(0,0, L)=\exp [2 \chi(\beta)] \exp \left[-2 \frac{\beta^{2}}{W_{\mathrm{ST}}^{2}(L)}\right] .
$$

The point detector will see the variation of the optical irradiance that is due to beam wander, and, moreover, the variation in scintillation index that is due to its dependence on its relative position in the beam.

Equation (13) sets the value of the scintillation index, assuming that the irradiance average is unity in each position on the beam taken one by one, but we have to deal with the whole beam at a time. The average of the irradiance may be written as

$$
\langle I(r, L)\rangle=\exp \left[-2 \frac{r^{2}}{W_{\mathrm{LT}}^{2}(L)}\right],
$$


and the scintillation index normalized in such a way that irradiance is unity at the beam center results in

$$
\sigma_{I, \mathrm{~Gb}}^{2}=\left(\sigma_{I}^{2}+\sigma_{I, r}^{2}\right)\langle I\rangle^{2},
$$

where Gb means Gaussian beam.

Apart from the effect of beam wander, it is known that the irradiance that is due to the scintillation phenomenon, at least in weak to moderate turbulence strength, follows a log-normal probabilitydensity function. ${ }^{24}$ Then the (partial) log amplitude is a normal random variable, and these relations are approximately satisfied:

$$
\begin{aligned}
& \langle\chi\rangle=-\sigma_{\chi}^{2}, \\
& \sigma_{\chi}^{2}=1 / 4 \ln \left(1+\sigma_{I, \mathrm{~Gb}}{ }^{2}\right),
\end{aligned}
$$

assuming that the mean irradiance is unity at the beam center.

So the simulation procedure is as follows:

(a) The second-order moment $\left\langle\beta^{2}\right\rangle$ is calculated by means of Eq. (11), and from Eq. (10) we obtain the variance of the displacements of the beam center along the transverse axes.

(b) We generate a sequence of normal random variables, $\beta_{x}$ and $\beta_{y}$, with zero mean and the appropriate variance.

(c) With each generation of a random pair $\left(\beta_{x}, \beta_{y}\right)$

(i) We obtain a random position $\beta$ of the beam center. With this value we calculate the off-axis term of the scintillation index from Eq. (20), and the average irradiance from Eq. (24), with $r=\beta$. The on-axis term, Eq. (16), must also be calculated, although it is invariant with the position of the beam center.

(ii) The variances of the irradiance fluctuations are obtained from Eq. (25).

(iii) The partial log-amplitude mean and variance are obtained now from Eq. (26). obtained.

(iv) A single instance of a $\chi$ random variable is

(d) With a large enough sequence of instances of $\beta$ and $\chi$ an irradiance sequence is obtained as

$$
I=\exp (2 \chi) \exp \left[-\frac{2 \beta^{2}}{{W_{\mathrm{ST}}}^{2}(L)}\right]
$$

at the expected position of the beam center, where the point detector is assumed to be located.

(e) Finally, we express the irradiance as a function of the total log amplitude, including the beamwander effects. From the random sequence of irradiance values obtained in the previous step, the total log amplitude or irradiance variance is calculated statistically.

\section{Numerical Results}

For all the calculations that we make in this section, a Kolmogorov spectrum and the index structure con-

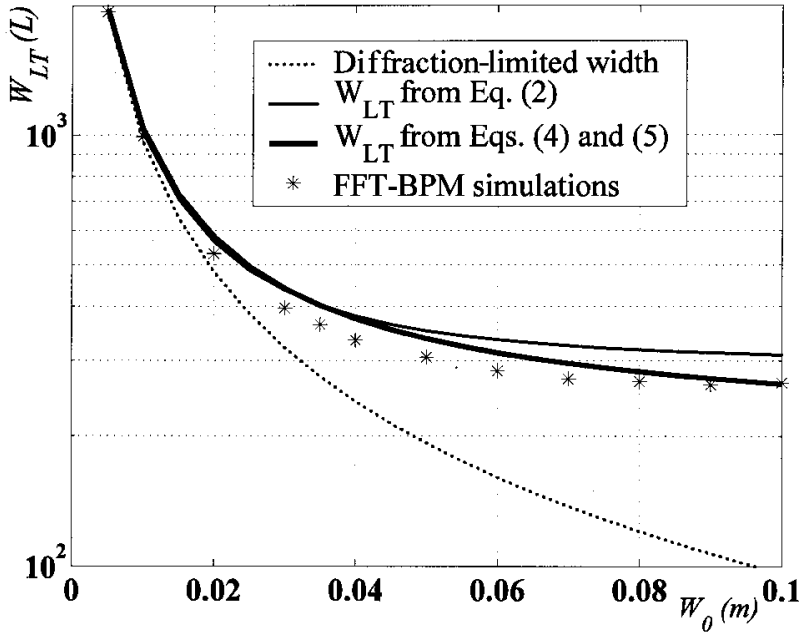

Fig. 1. Long-term beam spread at the reception plane situated in a geostationary orbit. Three methods were used for the calculation, as shown.

stant model $C_{n}{ }^{2}(h)$ given in Ref. 20 have been assumed. This model is

$$
\begin{aligned}
C_{n}^{2}(h)= & 0.00594(v / 27)^{2}\left(h \times 10^{-5}\right)^{10} \exp (-h / 1000) \\
& +2.7 \times 10^{-16} \exp (-h / 1500) \\
& +A \exp (-h / 100),
\end{aligned}
$$

with $A=1.7 \times 10^{-14} \mathrm{~m}^{-2 / 3}$ and $v=21 \mathrm{~m} / \mathrm{s}$.

We made several comparisons with the split-step numerical method [or fast-Fourier-transform beam propagation method (FFT-BPM)] to check the validity of the theoretical expressions that describe the beam effects in our particular conditions. We formulated the FFT-BPM in spherically diverging coordinates, and it uses $\sim 20$ fractal, $256 \times 256$ point, phase screens to simulate the atmospheric turbulence. ${ }^{25,26}$ The number of simulations was at least 300 for each calculated point. The phase screens were chosen to be not uniformly spaced; they were closer to one another at the beginning of the uplink path, where the turbulence is more intense. We assumed that the satellite was in a geostationary orbit, with $L=36 \times 10^{6} \mathrm{~m}$, and the limit of the atmosphere was taken at $20 \mathrm{~km}$. The wavelength was $\lambda=0.84$ $\mu \mathrm{m}$. The optical ground station was at sea level, and the analyses were performed for two elevation angles and for different waists for the laser beam, which we have taken as collimated.

In Fig. 1 the long-term beam width, in agreement with Eqs. (2), (4), and (5), is plotted as a function of the initial beam waist. Results are compared with those obtained by means of the FFT-BPM in spherically diverging coordinates. Figures 1-4 have been calculated for an elevation angle of $90^{\circ}$.

Figure 2 shows the behavior of the short-term beam width as a function of the initial beam waist. The second-order moment of the beam wander is plotted in Fig. 3. In both cases we again represented the results obtained with beam-propagation method sim- 


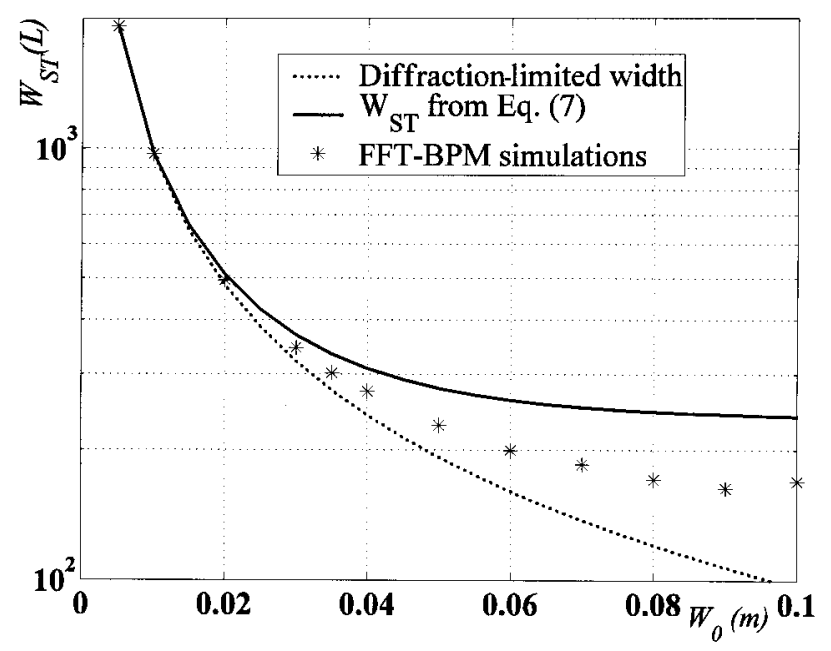

Fig. 2. Short-term beam width in a stationary orbit. The differences between the classic expression given by Yura ${ }^{22}$ and the FFTBPM simulations are apparent.

ulations. We obtained numerical short-term beam width results by removing the beam displacement in each simulation and averaging the beam irradiance. In Figs. 1-3 the analytical formulas and the simulations gave similar but slightly different results; the short-term beam waist was the parameter that differed most.

To prove the consistency of the analytical formulas and of the numerical method, we define an error term, which is readily deduced from Eq. (1):

$$
\varepsilon=\left|1-\frac{W_{\mathrm{ST}^{2}}^{2}+2\left\langle\beta^{2}\right\rangle}{W_{\mathrm{LT}}^{2}}\right| .
$$

In Fig. 4, error curves obtained for both analytical and numerical methods are shown. It seems that the beam propagation method maintains higher accuracy than the analytical expressions presented in the preceding sections. Anyway, it has to be said that these error curves have only a limited validity

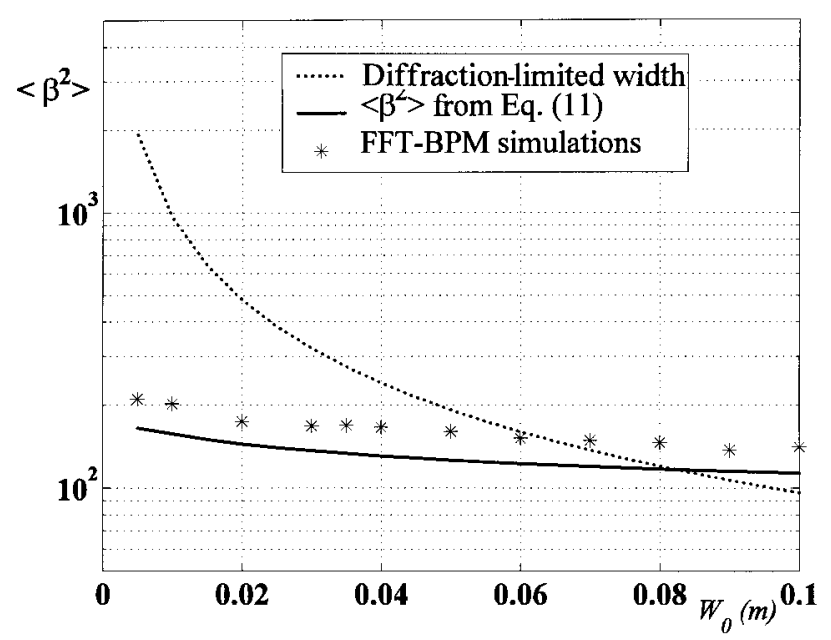

Fig. 3. Second-order moment of the beam wander.

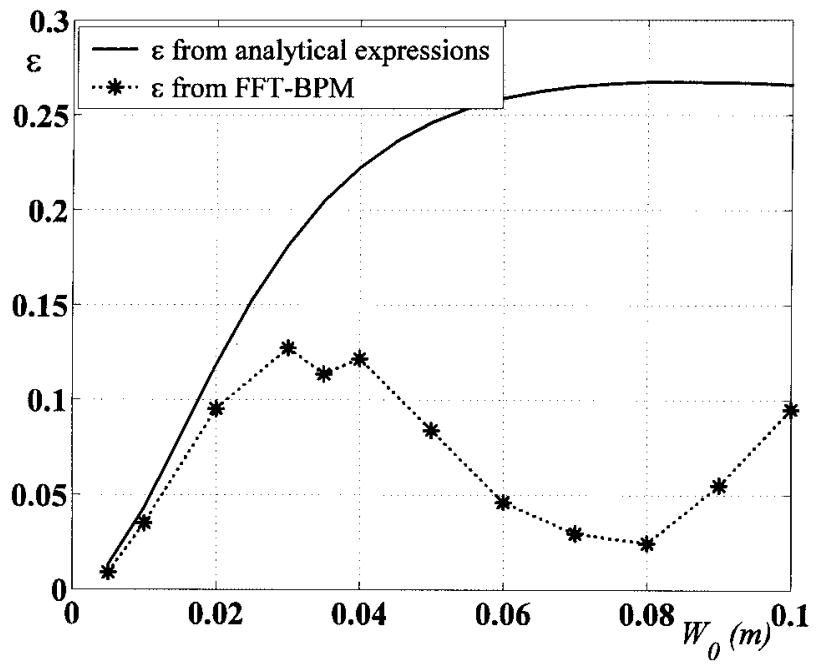

Fig. 4. Intrinsic error in the preceding calculations, following Eq. (29).

because throughout this paper and in particular in Eqs. (1) and (29) it is assumed that the beam maintains its Gaussian shape along the propagation in the turbulence, and this assumption is not exact.

In Fig. 5 some results obtained from the semianalytical procedure presented in Section 4 are shown. The log-amplitude variance is plotted versus the initial beam waist. Again, the results are compared with those worked out with the FFT-BPM. Calculations were performed for two values of the elevation angle. Dotted curves represent the on-axis scintillation, which is the only important contribution for small values of $W_{0}$, when the beam seems to be a spherical beam as viewed from the satellite.

It can be seen that the agreement of the two meth-

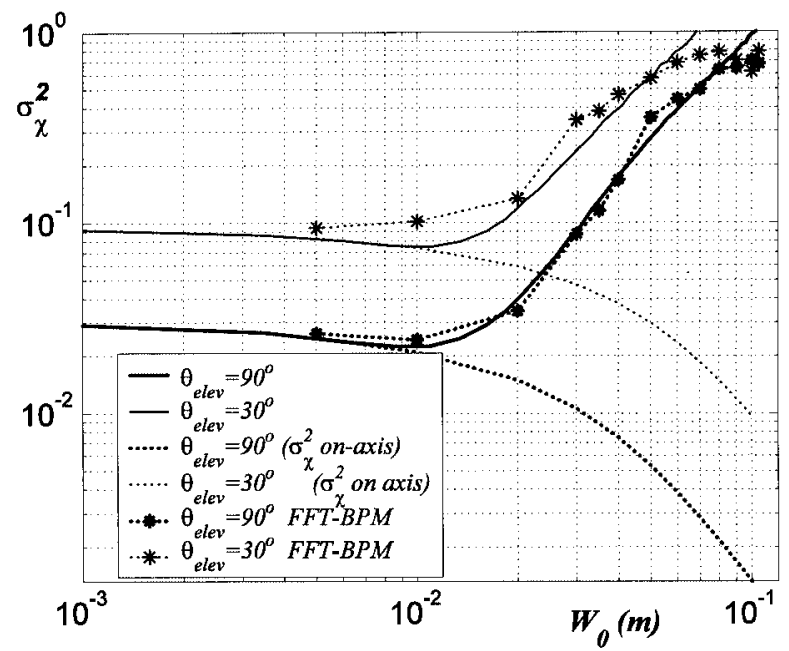

Fig. 5. Log-amplitude variance in the uplink of a geostationary satellite as a function of the initial waist of a collimated beam, obtained by the semianalytical method presented in this paper. The wavelength was $0.84 \mu \mathrm{m}$. The correspondence with the numerical calculations (the asterisk curves) is good enough until the appearance of the saturation effect. 


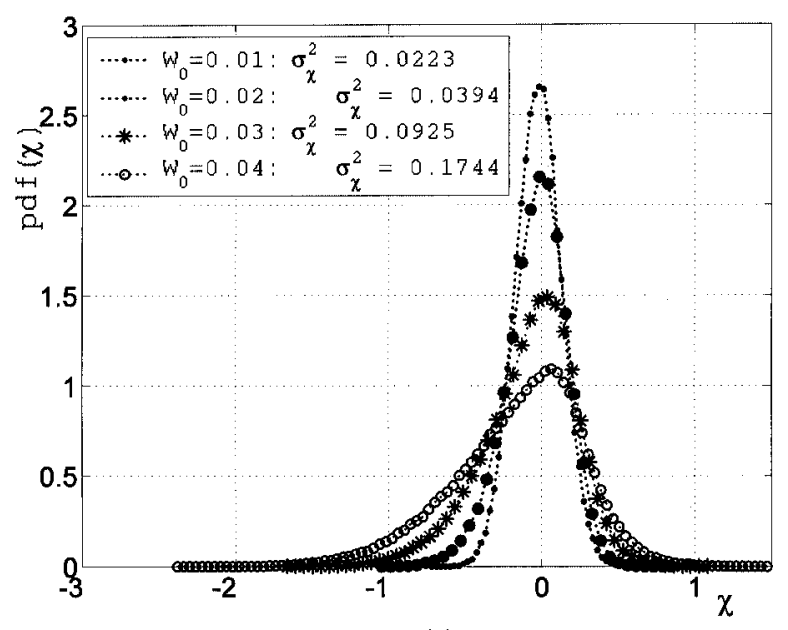

(a)

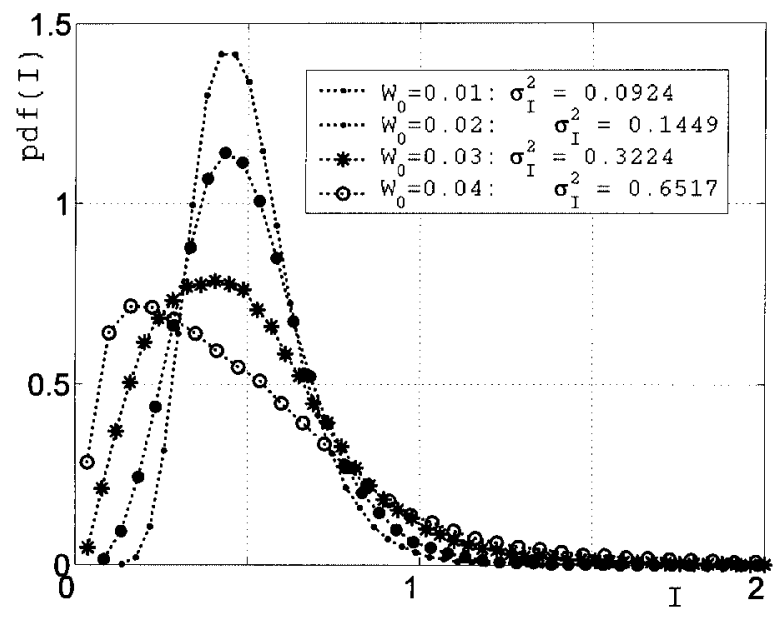

(b)

Fig. 6. (a) Pdf of the log amplitude, deduced from a high number of simulations with the semianalytical method presented in this paper. The normal value of the pdf holds only for small values of the log-amplitude variance. (b) Corresponding pdf of the irradiance. For small variances in irradiance it is a log-normal function, but it loses this characteristic when the influence of beam wander appears.

ods is good when there is weak turbulence. From the critical value of $\sigma_{\chi}{ }^{2} \sim 0.6$ the curve generated with the beam propagation method shows the saturation effect, whereas the approximate semianalytical method does not take this fact into consideration.

The probability density function (pdfs) of both the irradiance and the log amplitude are plotted in Fig. 6 for several values of the initial beam waist. Our analysis has shown that, for small values of the final variance, the $\chi$ pdf is a normal curve; but such is not the case, as could be expected, when the variance increases because of the effect of beam wander.

Finally, we can observe the different behavior of the log-amplitude variance as a function of wavelength (Fig. 7). The influence of the beam wander appears earlier as the wavelength becomes smaller, as could be expected.

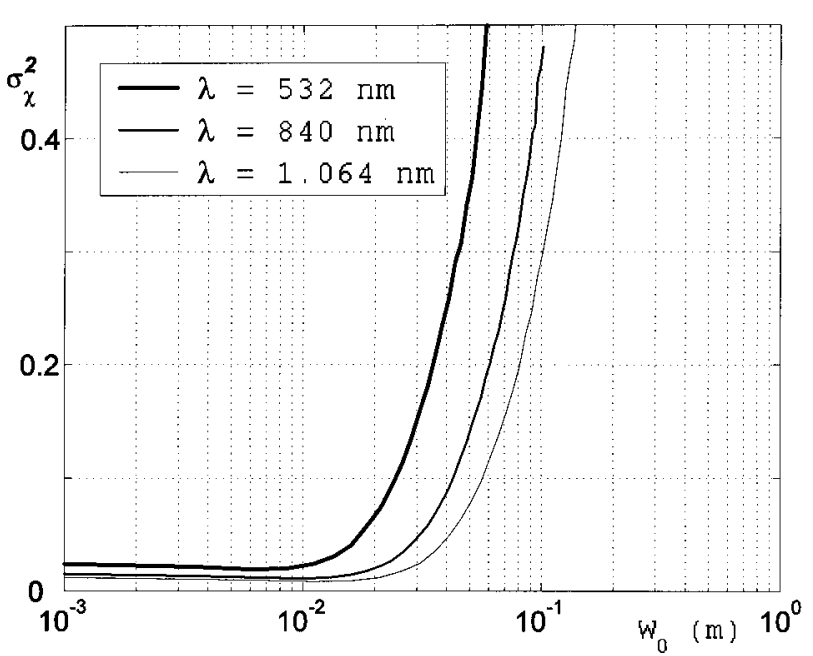

Fig. 7. Effect of beam wander on the uplink's log-amplitude variance as a function of beam waist and wavelength.

\section{Conclusions}

We have presented a new methodology with which to simulate the combined effect of scintillation and beam wander in the propagation of laser beams through atmospheric turbulence. Although this technique, in the version presented here, does not include the saturation effect, it provides enough good results in an important range of turbulence strengths and requires only a small computational effort. For example, the curves shown in Fig. 5 were generated in few minutes with a desktop computer, whereas the equivalent FFT-BPM simulations required 2 days on the same machine.

This study has been partially supported by European Space Agency contract 16021/02/NL/CK.

\section{References}

1. J. W. Strohbehn, "Line-of-sight wave propagation through the turbulent atmosphere," Proc. IEEE 56, 1301-1318 (1968).

2. V. I. Tatarski, The Effects of the Turbulent Atmosphere on Wave Propagation (Israel Program for Scientific TranslationNOAA, Jerusalem, 1971).

3. S. F. Clifford, G. R. Ochs, and R. S. Lawrence, "Saturation of optical scintillation by strong turbulence," J. Opt. Soc. Am. 64, 148-154 (1974).

4. A. M. Prokhorov, F. V. Bunkin, K. S. Gochelashvily, and V. I. Shishov, "Laser irradiance propagation in turbulent media," Proc. IEEE 63, 790-811 (1975).

5. R. L. Fante, "Electromagnetic beam propagation in turbulent media," Proc. IEEE 63, 1669-1692 (1975).

6. J. W. Strohbehn, ed., Laser Beam Propagation in the Atmosphere (Springer-Verlag, Berlin, 1978).

7. R. L. Fante, "Electromagnetic beam propagation in turbulent media: an update," Proc. IEEE 68, 1424-1443 (1980).

8. J. H. Churnside, "Aperture averaging of optical scintillations in the turbulent atmosphere," Appl. Opt. 30, 1982-1994 (1991).

9. I. I. Kim, H. Hakakha, P. Adhikari, E. Korevaar, and A. K. Majumdar, "Scintillation reduction using multiple transmitters," in Free-Space Laser Communication Technologies IX, G. S. Mercherle, ed., Proc. SPIE 2990, 102-113 (1997). 
10. L. C. Andrews and R. L. Phillips, Laser Beam Propagation through Random Media, Vol. PM53 of SPIE Press Monograph Series (SPIE Press, Bellingham, Wash., 1998).

11. L. C. Andrews, R. L. Phillips, C. Y. Hopen, and M. A. AlHabash, "Theory of optical scintillation," J. Opt. Soc. Am. A 16, 1417-1429 (1999).

12. J. H. Churnside and R. J. Lataitis, "Wander of an optical beam in the turbulent atmosphere," Appl. Opt. 29, 926-930 (1990).

13. R. A. Schmeltzer, "Means, variances and covariances for laser beam propagation through a random medium," Q. Appl. Math. 24, 339-354 (1966).

14. A. Ishimaru, "Fluctuations of a focused beam wave for atmospheric turbulence probing," Proc. IEEE 57, 407-414 (1969).

15. L. Andrews, Department of Mathematics, University of Central Florida, Orlando, Fla. (personal communication, 22 January 2004).

16. R. E. Hufnagel, "Propagation through atmospheric turbulence," in The Infrared Handbook, W. L. Wolfe and G. J. Zissis, eds. (U.S. Office of Naval Research, Washington, D.C., 1978), Chap. 6.

17. P. J. Titterton, "Power reduction and fluctuations caused by narrow laser beam motion in the far field," Appl. Opt. 12, 423-425 (1973).
18. T. Chiba, "Spot dancing of the laser beam propagated through the turbulent atmosphere," Appl. Opt. 10, 2456-2461 (1971).

19. D. H. Tofsted, "Outer-scale effects on beam-wander and angleof-arrival variances,” Appl. Opt. 31, 5865-5870 (1992).

20. L. C. Andrews, R. L. Phillips, and P. T. Yu, "Optical scintillation and fade statistics for a satellite-communication system," Appl. Opt. 34, 7742-7751 (1995).

21. H. T. Yura, C. C. Sung, S. F. Clifford, and R. J. Hill, "Secondorder Rytov approximation,” J. Opt. Soc. Am. 73, 500-502 (1983).

22. H. T. Yura, "Short-term average optical-beam spread in a turbulent medium," J. Opt. Soc. Am. 63, 567-572 (1973).

23. A. Belmonte, "Feasibility study for the simulation of beam propagation: consideration of coherent lidar performance," Appl. Opt. 39, 5426-5445 (2000).

24. J. W. Goodman, Statistical Optics (Wiley, New York, 2000).

25. R. G. Lane, A. Glindeman, and J. C. Dainty, "Simulation of a Kolmogorov phase screen," Waves Random Media 2, 209-224 (1992).

26. C. M. Harding, R. A. Johnston, and R. G. Lane, "Fast formulation of a Kolmogorov phase screen," Appl. Opt. 38, 21612170 (1999). 